\title{
Impact of Maternal Undernutrition on Hypothalamo-Pituitary-Adrenal Axis and Adipocyte Functions in Male Rat Offspring
}

\author{
Andrea N. Chisari, ${ }^{1,2}$ Andres Giovambattista, ${ }^{1,2}$ Mario Perello, ${ }^{1}$ and Eduardo Spinedi ${ }^{1}$ \\ ${ }^{1}$ Neuroendocrine Unit, Multidisciplinary Institute on Cell Biology, CONICET-CIC and ${ }^{2}$ School of Exact Sciences, \\ UNLP, La Plata, Argentina
}

Malnutrition induces profound deleterious effects on several metabolic and neuroendocrine functions. In the present study, we examined the impact of maternal food restriction, during gestation and lactation, on the metabolic-neuroendocrine function of their male offspring at 21 and $60 \mathrm{~d}$ of age. Well-nourished (WN) and undernourished (UN) pregnant rats were used, during gestation and lactation, until pups were weaned. Twenty-one-day-old $W N$ and $U N$ male pups were studied in basal and postinsulin conditions. Additional groups of weaned (WN and UN) male rats were fed either ad libitum (WN-WN and UN-WN) or in a restricted fashion (WN-UN and UN-UN) until experimentation at age $60 \mathrm{~d}$. Body weights of mothers and their male offspring were monitored. Basal and postinsulin plasma concentrations of several metabolic fuels were evaluated. Our results indicate that 21-dold UN male rats exhibited (vs their WN counterparts), decreased body weights, similar basal and postinsulin glycemia, similar basal plasma adrenocorticotropic hormone (ACTH) and corticosterone levels but diminished ACTH response to insulin treatment, and basal hypoleptinemia and significant insulin-induced leptin release. Finally, at $60 \mathrm{~d}$ of age, long-term UN (WN-UN and UN-UN) rats showed lower plasma (basal and postinsulin) glucose, and basal triglyceride levels than their counterparts (WN-WN and UN-WN). Sixty-dayold rats submitted to either food restriction protocol also showed a reduced hypothalamo-pituitary-adrenal axis response to insulin-induced hypoglycemia and basal hypoleptinemia, in spite of restoration of normal body weights. These results further indicate a clear metabolic-neuroendocrine dysfunction in male pups of UN mothers, with the abnormality partially present at weaning and deteriorated by adulthood, even after the recovery of normal body weight. Our study strongly supports the importance of the irreversibility of a dele-

Received November 3, 2000; Revised January 15, 2001; Accepted February 9, 2001

Author to whom all correspondence and reprint requests should be addressed: Dr. Eduardo Spinedi, Neuroendocrine Unit, IMBICE, cc 403, 1900 La Plata, Argentina. E-mail: esimbi@netverk.com.ar terious allostatic state resulting from fetal and early postnatal undernutrition.

Key Words: Adrenocorticotropic hormone; glucocorticoid; carbohydrate; leptin; foodrestriction.

\section{Introduction}

Cachexia, immune dysfunction, and other metabolic disturbances are linked to malnutrition of individuals with a poor prognosis after infection, injury, or many illnesses ( 1 , 2 ). Both malnutrition (3) and undernutrition (4) are accompanied by high levels of circulating glucocorticoid, which, in turn, can induce immune $(5,6)$ and other metabolic disturbances (4) that contribute to the pathophysiologic effects of different eating disorders.

Calorie and protein malnutrition may arise from a variety of conditions, and both are characterized by enhanced basal hypothalamic-pituitary-adrenal (HPA) axis activity $(3,4)$, However, several symptoms found in many cases of nutrition disturbances are connected with associated parasitic or bacterial infections, and, therefore, the relative contribution of calorie and protein malnutrition to enhanced glucocorticoid production has been controversial $(7,8)$. Nevertheless, it has been demonstrated that neuroendocrine-metabolic-immune dysfunction induced by severe undernutrition, starting after weaning, can be rapidly reversed by placing individuals on an ad libitum food intake program $(4,9,10)$, indicating that this serious allostatic state, characteristic of undeveloped countries, can be prevented by an improvement in socioeconomic conditions.

On the other hand, there is little information on the consequences of maternal undernutrition on the offspring's HPA axis and other metabolic functions. One study showed that in sheep abnormal development of the fetal HPA axis occurred as a consequence of nutrient restriction of mothers in early gestation, resulting in HPA axis hyporesponsiveness to hypoxemia (11). Whereas undernutrition during late gestation in rats has been reported to induce maternal adaptive mechanisms, which, in turn, could produce fetal endocrine pancreas dysfunction (12), poor nutrition of mothers during gestation and lactation was demonstrated to lead to 


\section{Table 1}

Body Weights of WN $(n=12)$ and UN $(n=20)$ Mothers Before (d 0) and on Different Days of Pregnancy ${ }^{a}$

\begin{tabular}{cll}
\hline Day & \multicolumn{1}{c}{ WN } & \multicolumn{1}{c}{ UN } \\
\hline 0 & $177.6 \pm 8.6$ & $174.7 \pm 3.7$ \\
6 & $190.7 \pm 10.4$ & $175.2 \pm 3.3$ \\
13 & $215.2 \pm 12.1^{b}$ & $179.5 \pm 3.6^{c}$ \\
20 & $251.3 \pm 13.3^{b}$ & $202.7 \pm 4.6^{b, c}$ \\
\hline
\end{tabular}

${ }^{a}$ Body weights (g) were recorded between 7:00 AM and 7:30 AM.

${ }^{b} p<0.05$ vs respective d 0 values.

${ }^{c} p<0.05$ vs $\mathrm{WN}$ values on the same day.

impaired pancreatic $\beta$-cell activity during adulthood (13). These findings clearly underscore the importance of maternal homeostasis for a normal adult life and for the development of appropriate metabolic-endocrine responses in acute/ chronic allostatic states.

The present study explored the effects of severe maternal undernutrition, during gestation and lactation, on HPA axis and adipocyte functions of the male offspring, at weaning and on d 60 of age. Experimentation was controlled against age-matched male rats born from well-nourished mothers (WN) submitted or not to long-term undernutrition after weaning.

\section{Results}

\section{Evolution of Body Weight of Mothers \\ During Pregnancy And Their Male Products as a Consequence of Restricted Food Intake}

The evolution of body weights in mothers as a consequence of food restriction is shown in Table 1. An arrest in body weight gain in pregnant rats was present, although not significantly different from their counterparts, at the end of the first week of pregnancy; however, food restriction significantly $(p<0.05)$ reduced body weight gain by the end of both the second and third weeks of pregnancy. Nevertheless, body weights of undernourished (UN) mothers on the day before partum were significantly $(p<0.05)$ higher than the respective $\mathrm{d} 0$ values. Restriction of food intake continued after the offsprings' birth (see Fig. 1, top, and Table 2); note that we did not record body weights of mothers because, in preliminary experiments, we found a high rate of litter mortality as a consequence of the additional stress induced by handling. The number of total weaned pups per mother was significantly $(p<0.05)$ reduced in UN rats (7.68 \pm 0.51 vs $10.36 \pm 0.72$ in WN mothers) but not as a consequence of a reduction in the number of weaned males per mother $(4.91 \pm 0.49$ and $3.95 \pm 0.33$ in $\mathrm{WN}$ and $\mathrm{UN}$ mothers, respectively).

Figure 2 (top) shows the results of body weights on different days of age in the four groups established. When day
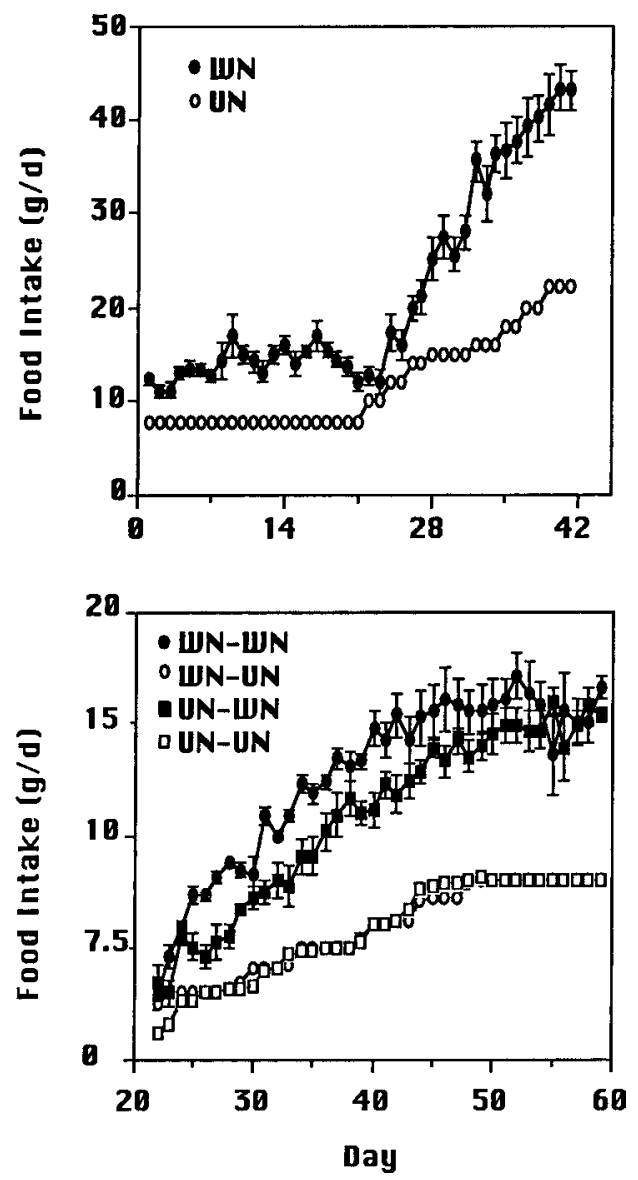

Fig. 1. (Top) Daily food intake of WN $(n=12)$ and UN $(n=20)$ mothers on different days of gestation (d 1-21) and lactation (d 21-41). (Bottom) Food intake of male rats from several groups: $\mathrm{WN}$ mothers during gestation and lactation-WN from weaning up to d 59 (WN-WN), WN-UN from d 22 up to d 59 (WN-UN), UN mothers during gestation and lactation-WN from weaning up to d 59 (UN-WN), and UN-UN ( $n=13-17$ rats/group).

Table 2

Daily Food Allowance in Lactating Mothers ${ }^{a}$

\begin{tabular}{lllr}
\hline & Group of mothers & $\begin{array}{l}\text { WN } \\
(\%)\end{array}$ & $\begin{array}{r}\text { UN } \\
(\%)\end{array}$ \\
\hline Lactation & Days 1-3 & 100 & $83-97$ \\
& Days 4-5 & 100 & $69-75$ \\
& Days 6-7 & 100 & $66-70$ \\
& Days 8-10 & 100 & $55-59$ \\
& Days 11-13 & 100 & $44-53$ \\
& Days 14-15 & 100 & $44-49$ \\
& Days 16-17 & 100 & $48-51$ \\
Weaned group & Days 18-20 & 100 & $50-53$ \\
\hline
\end{tabular}

${ }^{a}$ Days correspond to d 21-41 in Fig. 1, top. WN mothers received food available ad libitum during $24 \mathrm{~h} / \mathrm{d}$ (for daily food intake, see Fig. 1, top), and UN mothers were provided with the indicated percentage of the amount ate per WN mother, at a similar period of time. 

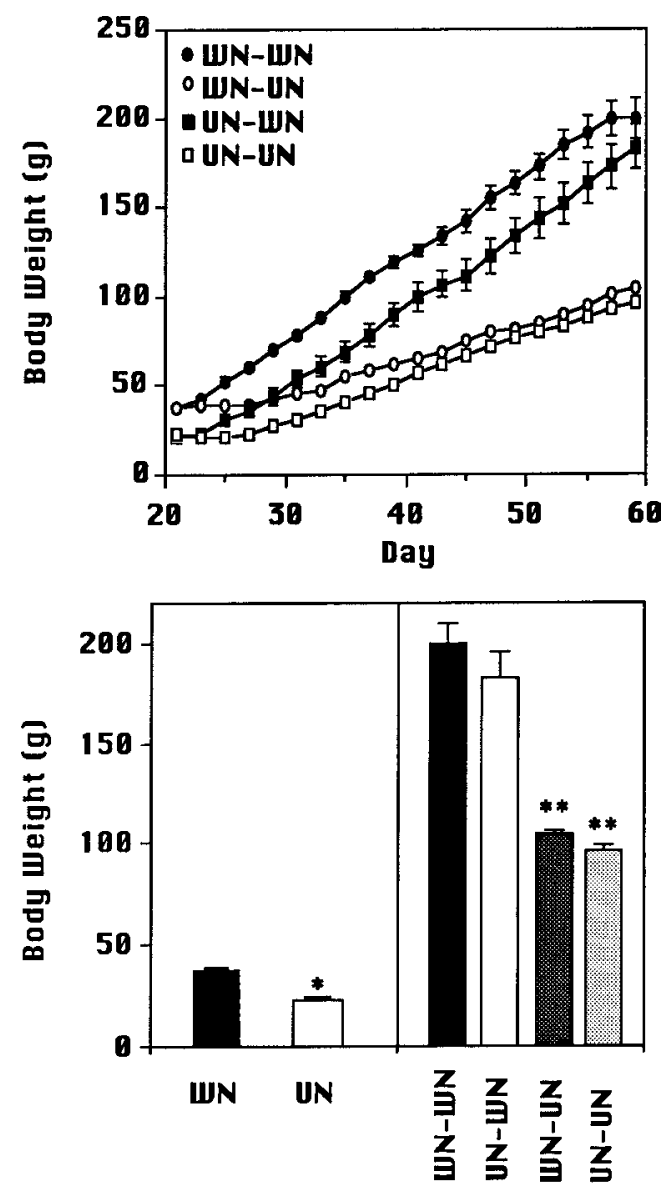

Fig. 2. Evolution of body weights in several experimental groups, recorded day by day, after weaning (d 21) and up to d 59 of age (top, $n=12-17$ rats/group). Body weights of male rats submitted to experiment 1 (age $21 \mathrm{~d}$, lower left) and experiment 2 (age $59 \mathrm{~d}$, lower right) (values are the same depicted at top for $\mathrm{d} 21$ and 59 of age). ${ }^{*} p<0.05$ vs WN values; $* * p<0.05$ vs WN-WN and UN$\mathrm{WN}$ values.

and status of the mother (WN and $\mathrm{UN}$ ) were compared in rats eating ad libitum after weaning, body weights were significantly $(p<0.05)$ lower in rats born from UN than from WN mothers up to $\mathrm{d} 49$; similarly, but in groups submitted to the food restriction protocol, body weights were significantly $(p<0.05)$ lower in rats born from UN than from WN mothers up to $\mathrm{d} 47$. On the other hand, when comparing the effect of food restriction on weaned male pups from the same mother group (WN-UN vs WN-WN and UN-UN vs UN-WN), data analysis indicates that body weights were significantly $(p<0.05)$ different from d 25 of age.

As depicted in Fig. 2, male pups weaned from UN mothers displayed a significantly $(p<0.05)$ lower body weight than their normal counterparts. On d 59 of age, no significant differences in body weights were found between male rats ad libitum born from both $\mathrm{WN}$ and $\mathrm{UN}$ mothers (WNWN and UN-WN groups), nor between food-restricted males born from both groups of mothers (WN-UN and UN-
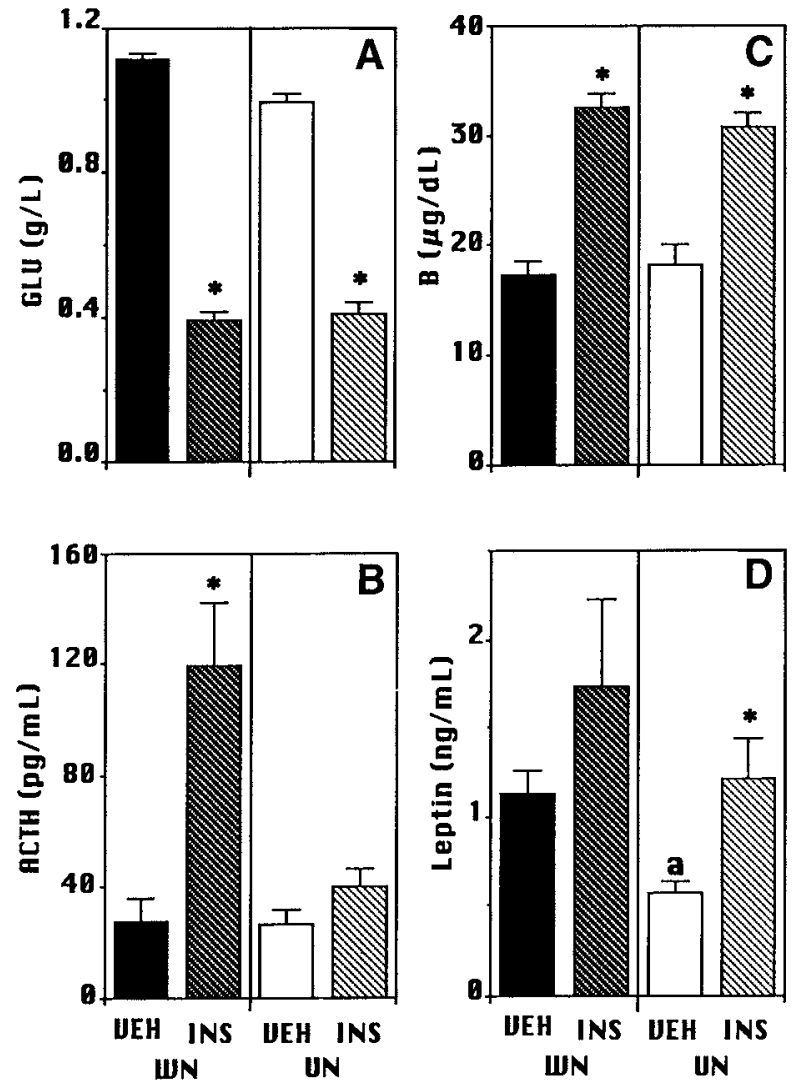

Fig. 3. (A) Plasma glucose, (B) ACTH , (C) glucocorticoid (B), and (D) leptin levels $45 \mathrm{~min}$ after ip administration of vehicle (VEH) or insulin (INS) in 21-d-old male pups of WN and UN mothers ( $n=6-9$ rats/group). ${ }^{*} p<0.05$ vs respective VEH values; ${ }^{a} p<0.05$ vs $\mathrm{VEH}$ values in WN pups.

UN rats); however, body weights of food-restricted males (WN-UN and UN-UN groups) were significantly $(p<0.01)$ lower than those of rats born from both groups of mothers (WN and UN) but fed ad libitum until d 59.

\section{Insulin-Induced Hypoglycemia \\ in 21-d-old Rats Born from WN and UN Mothers}

Basal plasma glucose, ACTH, and corticosterone (B) concentrations were not modified by undernutrition of mothers (Fig. 3A-C). Administration of insulin to male pups at wean-ing was able to significantly $(p<0.05)$ reduce plasma glucose levels to below the respective baselines (vehicle values) in WN and UN pups 45 min posttreatment (Fig. 3A). Insulin-elicited ACTH release was significantly $(p<0.05)$ higher than the respective baseline only in WN pups (Fig. 3B); however, insulin-induced hypoglycemia was accompanied by an increase in circulating glucocorticoid over $(p<0.05)$ the respective baseline (Fig. 3C) in both groups examined. Finally, decreased body weight in pups from UN mothers was correlated with basal hypoleptinemia $(p<0.05$ vs WN values) (Fig. 3D). However, when ratios plasma leptin level:body weight per 100 were compared, no significant differences were found in basal condition $(4.39 \pm 0.65$ 

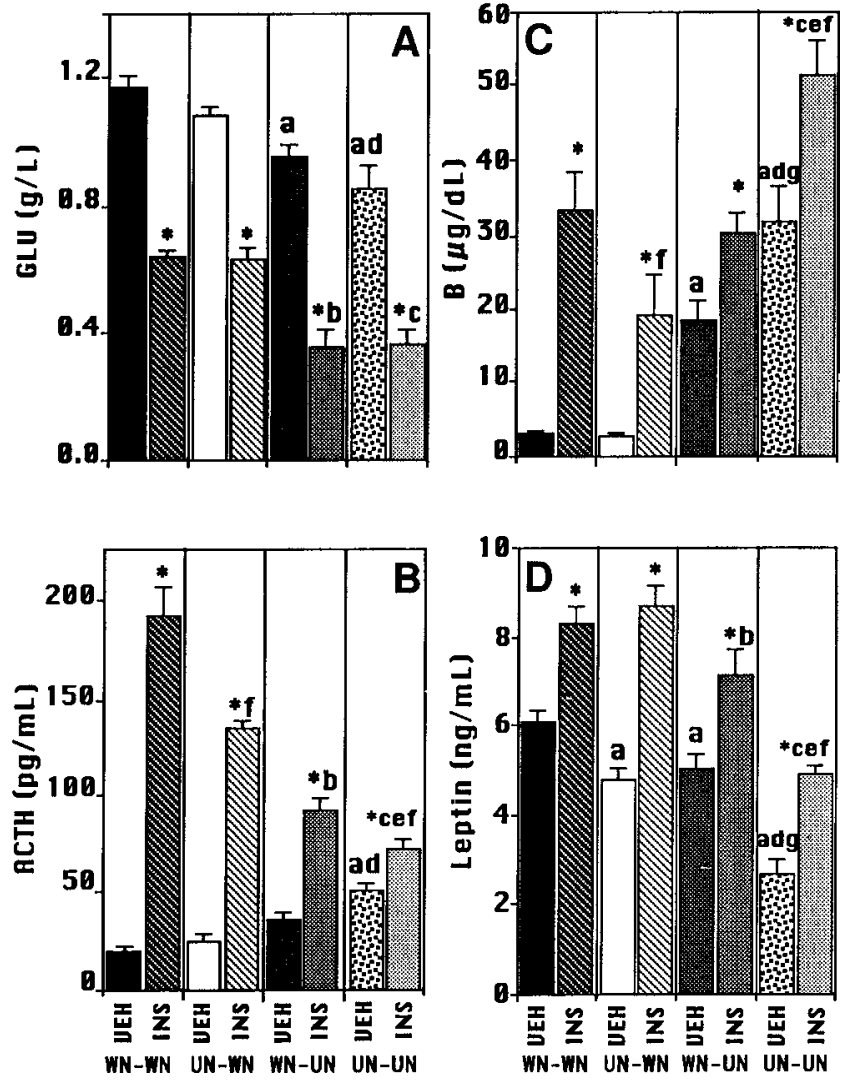

Fig. 4. (A) Circulating glucose, (B) ACTH, (C) glucocorticoid, and (D) leptin concentrations in basal (vehicle [VEH]) and postinsulin INS conditions in 60-d-old male rats of different groups ( $n=6-9$ rats/group). $* p<0.05$ vs respective VEH values; ${ }^{a} p<0.05$ vs VEH values in WN-WN rats; ${ }^{b} p<0.05$ vs INS values in WN-WN rats; ${ }^{c} p<0.05$ vs INS values in UN-WN rats; ${ }^{d} p<0.05$ vs VEH values in UN-WN rats; ${ }^{e} p<0.05$ vs INS values in WN$\mathrm{UN}$ rats; ${ }^{f} p<0.05$ vs INS values in WN-WN rats; ${ }^{g} p<0.05$ vs INS values in UN-UN rats.

and $3.66 \pm 0.51$ in $\mathrm{WN}$ and $\mathrm{UN}$ pups, respectively). Although the administration of insulin increased the mean of plasma leptin levels in both groups over the respective baseline, this increase was statistically significant $(p<0.05)$ only in UN pups (see also Fig. 3D) (plasma leptin level:body weight $=6.08 \pm 1.73$ in $\mathrm{WN}[p>0.05$ vs baseline $]$ and 6.86 \pm 1.31 in $\mathrm{UN}[p<0.05$ vs baseline] pups). In summary, different metabolic responses (ratio of stimulated:basal conditions) to insulin treatment were similar in both groups for hypoglycemia and glucocorticoid-secreting cells, higher in WN than UN pups for ACTH secretion, and lower in WN than in UN pups for adipose tissue.

\section{Effects of Food Restriction}

\section{for Mothers and Male Rats After Weaning on Metabolic-Neuroendocrine Function at 60 of Age}

Basal plasma glucose levels were similar in rats fed ad libitum after weaning, regardless of mothers (WN-WN and UN-WN groups); conversely, chronic food restriction in rats after weaning, born from either WN or UN mothers, induced basal hypoglycemia ( $p<0.05$ vs baselines in rats
Table 3

Plasma Triglyceride and Total Protein Concentrations, 45 min After Single ip Administration of Vehicle or Insulin, in Several Groups of 60-d-old Male Rats ( $n=6-9$ rats/group)

\begin{tabular}{lccc}
\hline Group & Treatment & $\begin{array}{c}\text { Triglyceride } \\
(\mathrm{g} / \mathrm{L})\end{array}$ & $\begin{array}{c}\text { Total protein } \\
(\mathrm{g} / \mathrm{L})\end{array}$ \\
\hline WN-WN & Vehicle & $1.19 \pm 0.11$ & $7.77 \pm 0.47$ \\
& Insulin & $0.98 \pm 0.14$ & $7.64 \pm 0.48$ \\
WN-UN & Vehicle & $0.61 \pm 0.09^{a}$ & $6.66 \pm 0.57$ \\
& Insulin & $0.28 \pm 0.05^{b}$ & $6.54 \pm 0.32$ \\
UN-WN & Vehicle & $1.18 \pm 0.23$ & $7.51 \pm 0.43$ \\
& Insulin & $1.29 \pm 0.17$ & $5.95 \pm 0.21^{b}$ \\
UN-UN & Vehicle & $0.58 \pm 0.09^{a}$ & $6.81 \pm 0.75$ \\
& Insulin & $0.48 \pm 0.03$ & $7.46 \pm 0.31$ \\
\hline
\end{tabular}

${ }^{a} p<0.05$ vs vehicle values in $\mathrm{WN}-\mathrm{WN}$ and $\mathrm{UN}-\mathrm{WN}$ rats.

$b_{p}<0.05$ vs respective vehicle values.

fed ad libitum after birth) (Fig. 4A). In all groups studied, insulin treatment induced significant $(p<0.05)$ hypoglycemia (vs the respective baseline). The pattern of insulin-induced hypoglycemia was characterized by lower $(p<0.05)$ plasma glucose levels in WN-UN and UN-UN groups than in WN-WN and UN-WN, respectively. However, plasma glucose levels postinsulin were similar in both groups of rats fed ad libitum after birth (WN-WN and UN-WN) and in both groups of UN rats after birth (WN-UN and UNUN). We found that there was no impact of undernutrition in mothers on basal triglyceride levels and that chronic undernutrition of weaned rats induced basal hypotriglyceridemia (Table 3). Although insulin treatment failed to modify basal plasma triglyceride levels in $\mathrm{WN}-\mathrm{WN}, \mathrm{UN}-\mathrm{WN}$, and UNUN groups, it significantly $(p<0.05)$ reduced (vs baseline values) triglyceridemia in WN-UN rats (Table 3 ). Neither protocol of food restriction modified basal plasma total protein levels, and the administration of insulin significantly $(p<0.05$ vs the respective basal) reduced this fuel only in $\mathrm{UN}-\mathrm{WN}$ rats (Table 3 ).

Figure 4B shows the results of basal and postinsulin plasma ACTH levels in several groups. Only severe undernutrition (UN-UN rats) induced a significant $(p<0.05)$ enhancement of basal ACTH secretion. Insulin-elicited ACTH secretion was significant ( $p<0.05$ vs the respective baseline) in all groups studied. However, insulin-stimulated ACTH release (ratio of stimulated:basal) was graded and followed a group order: $\mathrm{WN}-\mathrm{WN}>\mathrm{UN}-\mathrm{WN}>\mathrm{WN}-\mathrm{UN}>\mathrm{UN}-\mathrm{UN}$. Figure 4C shows circulating glucocorticoid levels, in basal and postinsulin conditions, in different groups. Rats eating ad libitum after weaning, regardless of the food intake of their mothers, displayed low basal plasma corticosterone levels (WN-WN and UN-WN groups). Chronically undernourished rats (WN-UN and UN-UN groups) showed significantly $(p<0.05)$ higher basal corticosterone levels than their WN counterparts, although basal plasma corticoster- 


\section{Table 4}

Plasma Leptin Level:Body Weight Ratios (per 100), in Basal (Vehicle) and Postinsulin Conditions, in Different Groups of 60-d-old Male Rats ( $n=6-9$ rats/group)

\begin{tabular}{lll}
\hline Group & \multicolumn{1}{c}{ Vehicle } & \multicolumn{1}{c}{ Insulin } \\
\hline WN-WN & $3.21 \pm 0.18$ & $4.11 \pm 0.45$ \\
WN-UN & $5.02 \pm 0.44^{a}$ & $7.21 \pm 0.52^{b, c}$ \\
UN-WN & $2.71 \pm 0.22$ & $4.64 \pm 0.35^{b}$ \\
UN-UN & $3.02 \pm 0.42$ & $5.28 \pm 0.41^{b}$ \\
\hline
\end{tabular}

\footnotetext{
${ }^{a} p<0.05$ vs basal WN-WN, UN-WN, and UN-UN values.

$b_{p}<0.05$ vs respective basal values.

${ }^{c} p<0.05$ vs insulin values in $\mathrm{WN}-\mathrm{WN}, \mathrm{UN}-\mathrm{WN}$, and $\mathrm{UN}-\mathrm{UN}$ groups.
}

one levels were significantly $(p<0.05)$ higher in UN-UN than in $\mathrm{WN}-\mathrm{UN}$ rats. Insulin treatment enhanced $(p<0.05)$ plasma corticosterone levels (over the respective baseline) in all groups studied. Adrenal response (stimulated:basal) to insulin treatment was similarly group ordered: $\mathrm{WN}-\mathrm{WN}$ $=\mathrm{UN}-\mathrm{WN}>\mathrm{WN}-\mathrm{UN}=\mathrm{UN}-\mathrm{UN}$.

Finally, basal plasma leptin levels were significantly ( $p$ $<0.05$ vs WN-WN values) lower in all groups of rats submitted to either food restriction protocol (Fig. 4D), although the lowest basal plasma leptin levels were found in UN-UN rats. Insulin treatment induced a significant $(p<0.05$ vs the respective baseline) leptin secretion in all groups, with the adipocyte response (stimulated:basal) following this order: $\mathrm{UN}-\mathrm{UN}>\mathrm{UN}-\mathrm{WN}=\mathrm{WN}-\mathrm{UN}>\mathrm{WN}-\mathrm{WN}$. When basal plasma leptin level:body weight ratios were expressed, this parameter was significantly $(p<0.05)$ higher in WN-UN rats than in the remaining groups (Table 4). Ratios increased, over the respective baselines, postinsulin; however, these increases were statistically significant $(p<0.05)$ only in rats submitted to either food restriction protocol (Table 4).

\section{Discussion}

We determined that mothers severely undernourished during pregnancy displayed an arrest in body weight gain, without modification of the normal length of gestation. This observation is in accord with earlier results showing that severe food restriction in mothers during the second or third, but not the first, week of gestation induced a significant arrest in body weight gain (12). Interestingly, when restricted food intake of mothers continued throughout lactation, the total number of weaned pups per UN dam was significantly lower (vs WN dam) owing to a decrease in the number of female, but not male, pups. We also observed decreased body weight in 21-d-old male pups from UN mothers, which correlated well with low basal circulating levels of leptin (14). An indicative fact for clear adaptation of these pups to basal condition was that no changes were noticed in the basal plasma levels of glucose, ACTH, and glucocorticoid; however, alteration in the circadian rhythm of glucocorti- coid secretion owing to undernutrition should not be excluded (15). Although insulin-induced hypoglycemia and glucocorticoid output were similar in both groups of male pups (from WN and UN mothers), ACTH plasma levels 45 min postinsulin were significantly lower in 21-d-old UN than in age-matched WN male pups. This fact could be owing to changes in the maturation of the hypothalamopituitary unit, resulting, as described in UN female pups at weaning (16), in a shifting to the left in the response of this unit, as a consequence of maternal stress (17) owing to long-term undernutrition. A significant ( $p<0.05$ vs baseline) stimulatory effect of insulin administration on plasma leptin levels (18) was found only in 21-d-old UN pups of both sexes (present results and ref. 16). Note that body weight and basal circulating leptin levels were lower in UN than in WN pups; however, insulin had an effect (19) in UN pups, mimicking plasma leptin levels in pups born from normally fed mothers.

In the second set of experiments we determined that body weights and plasma levels of glucose, triglyceride, total protein, $\mathrm{ACTH}$, and glucocorticoid were similar in 59-dold male (and female) (data not shown) rats eating ad libitum after weaning, regardless of the nutritional status of their mothers during gestation and lactation. Contrary to reports on maternal protein deficiency (13), during gestation and lactation, we found that food restriction in mothers during the same critical periods had no impact on the recovery of appropriate body weight of their 59-d-old products when fed ad libitum after weaning, regardless of the sex (present results and data not shown). However, when mothers were fed with a protein-deficient diet only during gestation, offspring recovered normal body weight at adulthood (20). These observations, taken together, could indicate that postnatal nutrition seems to be the critical period determining adult normal body weight, and that nutrient qualitative changes in food composition rather than the amount of food consumption could result in the most important factor predicting normal body weight at adult life. Interestingly, mild basal hypoleptinemia was found in male (and female) $(6.01 \pm 0.24$ vs $5.09 \pm 0.19$ in $\mathrm{WN}-\mathrm{WN}$ and $\mathrm{UN}$ $\mathrm{WN}$, respectively; $p<0.05$ ) products that were born from UN mothers but that ate ad libitum up to $60 \mathrm{~d}$ of age. This observation led us to speculate that while good nutrition after weaning would be an important determinant of normal body weight during adulthood, undernutrition during the fetal-postnatal period could severely affect the hypothalamic-peripheral circuit responsible for control in feeding behavior $(21,22)$.

Although starting from different body weights, a similar arrest in body weight gain, from weaning up to $\mathrm{d} 59$ of age, occurred in long-term UN male (and female) (data not shown) rats born from either group of mothers. Indeed, 60d-old male products from both groups of mothers (WN-UN and UN-UN) share several characteristics in basal condition, such as hypoglycemia, hypotriglyceridemia, somewhat 
enhanced plasma ACTH levels, hypercorticosteronemia, and hypoleptinemia. We previously reported that chronically undernourished (with a similar protocol of food intake) weaned female rats, born from WN mothers, were hypoglycemic and hypotriglyceridemic, with normal total protein concentration in plasma, in basal conditions (4). Although no changes in basal plasma glucose levels after 35\% restriction in food intake during 1 mo have been reported (23), that $35 \%$ restriction in food intake was accompanied by a $30 \%$ reduction in body weight in contrast to the $50 \%$ reduction in body weight induced by our design. Regarding long-term undernutrition-induced hypotriglyceridemia, which was found in rats from both sexes (present results and data not shown), our findings coincide with previous results indicating that chronic under-nutrition causes, as a compensatory factor for the allostatic state (24), a decrease in plasma levels of these lipids (25) to further preserve other metabolic pathways, such as protein metabolism (4).

When several responses to insulin administration were studied in different groups of 60-d-old WN rats, we found that, on the one hand, male products from both groups of mothers (WN and UN) attained a similar decrease in plasma glucose levels, a severalfold increase in plasma ACTH and corticosterone values (although both hormone levels were lower in products from UN than WN mothers), and an enhancement in the concentration of plasma leptin (similar results were found in female products; data not shown). These results clearly indicate that undernutrition of mothers had a serious deleterious impact on both neuroendocrine (HPA axis) and adipocyte responses, which cannot be overridden by the recovery of normal body weight. In fact, owing to similar insulin-induced hypoglycemia, lower pituitary, adrenal, and adipose tissue responses in UN-WN than in $\mathrm{WN}-\mathrm{WN}$ rats have taken place in order to counteract allostasis (24). On the other hand, insulin-treated male products born from WN and UN mothers and long-term undernourished after weaning displayed similar hypoglycemia, although more pronounced $(p<0.05)$ than products that ate ad libitum; significant ACTH responses, although lower $(p<0.05)$ than products that ate ad libitum, as well as marked glucocorticoid release in plasma; and an enhancement in circulating leptin levels. However, note that longterm food restriction in products born from UN, but not from WN, mothers resulted in an enhancement in basal plasma ACTH levels (similar findings were noticed in females, data not shown). It has been reported that protein malnutrition (3), but not chronic undernutrition (4), in rats born from $\mathrm{WN}$ mothers induces an increase in the drive of ACTH synthesis. Our data indicate that long-term food restriction did have an impact on pituitary function in male offspring born from UN but not from WN mothers.

It has been reported that the hypothalamic expression of corticotropin-releasing hormone ( $\mathrm{CRH})$, an anorectic signal (26), the physiologic regulator of HPA axis function
(27), is reduced, probably as a compensatory mechanism, after both food restriction and food deprivation (28). The precise mechanisms involved in the $\mathrm{CRH}$-dependent anorectic effects remain unclear. It has been postulated that food deprivation in lean rats induces a decrease in $\mathrm{CRH}_{2}-\mathrm{R}$ mRNA expression in the ventromedial nucleus of the hypothalamus (29), thus pointing to an effect on the CRH regulatory activity of energetic balance (30) rather than HPA axis function (27,31); however, there seems to be a cooperative involvement of both $\mathrm{CRH}_{1}-\mathrm{R}$ and $\mathrm{CRH}_{2}-\mathrm{R}$ on feeding behavior (32). In addition, a compensatory role of the hypothalamic vasopressinergic system on pituitary-adrenal activity $(33,34)$ could be developed to maintain appropriate ACTH and glucocorticoid release after chronic undernutrition; in fact, selective enhancement of the central vasopressinergic pathway, in $\mathrm{CRH}_{1}$-R-deficient mice recently has been postulated as a compensatory mechanism for HPA axis function (35).

A relation between a high-fat diet in mothers and HPA axis function of their products has been described $(36,37)$. It has also been reported that increased dietary fat content modifies both milk lipid levels of lactating mothers (38) and circulating leptin levels of their products (39). It has been further suggested that both the rise in 10-d-old pups and the decrease in 35-d-old offspring of circulating leptin levels could be, at least partially, responsible for decreased and increased, respectively, HPA axis responses to etherinduced stress (39).

Our results add valuable information on the body'sdefense mechanisms necessary for survival in negative energy balance conditions (40). It is important to remark that the deleterious effect of undernutrition, specifically during gestation and lactation, appears to be irreversible, even after recovery of normal body weight.

\section{Materials and Methods}

\section{Animals and Experimental Designs}

Controlled pregnant (determined by the presence of sperm in the vaginal smears after mating with an adult male for $24 \mathrm{~h}$ ) Fisher 344/N rats were individually housed in plastic cages (lights on 7:00 AM to 7:00 PM; $20-22^{\circ} \mathrm{C}$ ) and daily provided (before lights on) with standard rat pellets either ad libitum (WN) or in a restricted fashion $(8 \mathrm{~g} / \mathrm{d}$ throughout gestation) (UN) (Fig. 1, top, shows the results of food intake by gestating mothers of both groups.) The evolution of body weight was monitored at the end of the first and second weeks of gestation as well as the day before partum (Table 1). Mothers continued eating either ad libitum (WN) or in a restricted fashion (UN) (see also Fig. 1, top, and Table 2) until the pups were weaned. All rats received water ad libitum throughout the entire experiment. Experimentation was approved by our Institutional Animal Care Committee. 


\section{Experiment 1}

On d 21 of age (between 7:00 AM and 8:00 AM), pups per mother were counted and sex were determined, and body weights of the male pups recorded. Immediately afterward (between 8:00 AM and 8:30 $\mathrm{AM}$ ), male pups were injected intraperitoneally with either $50 \mu \mathrm{L}$ of sterile saline solution alone (vehicle) or containing insulin ( $1 \mathrm{IU} / \mathrm{kg}$ of body weight) (Actrapid; Nordisk AG, Switzerland (41).

\section{Experiment 2}

Weaned male pups, from WN and UN mothers, were housed in individual cages (lights on 7:00 AM to 7:00 PM) and provided for $24 \mathrm{~h}$ with water and standard rat pellets ad libitum. Between d 22 and 59, rats were submitted to the food-restricted protocol (daily food allowance provided during the end of the afternoon): $3 \mathrm{~g} / \mathrm{d}$ between $\mathrm{d} 21$ and 26 , $4 \mathrm{~g} / \mathrm{d}$ between $\mathrm{d} 27$ and 32, $5 \mathrm{~g} / \mathrm{d}$ between $\mathrm{d} 33$ and 37, $6 \mathrm{~g} / \mathrm{d}$ between $\mathrm{d} 38$ and $40,7 \mathrm{~g} / \mathrm{d}$, between $\mathrm{d} 41$ and 43 , and $8 \mathrm{~g} / \mathrm{d}$ between $\mathrm{d} 41$ and 59 (WN-UN and UN-UN groups). Additional groups of weaned male $\mathrm{WN}$ and $\mathrm{UN}$ pups were kept on ad libitum feeding up to experimental d 60, constituting the WN-WN and UN-WN groups, respectively. Body weight, and food and water intake were monitored daily (during the end of the afternoon; Fig. 1, bottom, shows the results of food intake by rats from different groups: WNWN, UN-WN, WN-UN, and UN-UN). Between 8:00 AM and 8:30 AM of $\mathrm{d} 60$, rats of different experimental groups were treated intraperitoneally with $50 \mu \mathrm{L}$ of vehicle alone or containing insulin, as described in experiment 1 .

Vehicle- or insulin-treated rats were returned to their home cages, without food, and killed by decapitation 45 min after treatment on d 21 (experiment 1) or on d 60 (experiment 2). Trunk blood was collected in plastic tubes containing $20 \mathrm{mg}$ of EDTA and kept in an ice bath until centrifuged. Because of the blood volume collected, plasma ACTH, glucocorticoid, leptin, and glucose were determined in rats from both experiments, and in individuals from experiment 2, plasma triglyceride and total protein concentrations were also measured.

\section{Metabolite Determinations}

Plasma total protein concentrations were measured by the classic Lowry's method. Plasma triglyceride and glucose concentrations were determined by enzymatic assays from Wiener Laboratories, Argentina. Circulating ACTH and corticosterone (B) concentrations were determined by specific immunoradiometric- and radioimmunoassays (RIAs), respectively, as previously described (42). The intraassay coefficients of variation (CVs) were 2 to 3 and $4-6 \%$ for $\mathrm{ACTH}$ and corticosterone, respectively; the interassay CVs were 58 and $10-12 \%$ for ACTH and corticosterone, respectively.

Plasma leptin concentrations were determined by an RIA developed in our laboratory and validated, for rat and mouse leptin, against a commercial kit (cat. no. RL-83K;
Linco Research, St. Charles, MO). This method was similar to several other RIAs developed in our laboratory (43). Briefly, synthetic murine leptin (PrePro Tech) was used for labeled peptide and standards as well as for the development of antileptin serum. Leptin was labeled $\left(\mathrm{Na}^{125} \mathrm{I}\right)$ by the chloramine $\mathrm{T}$ method and purified by elution, after loading the radioiodination mixture into a Sephacryl S-300 column (Sigma, St. Louis, MO) $(1.5 \times 60 \mathrm{~cm})$ with a sodium phosphate $(0.05 M)$, bovine serum albumin (BSA) $(2 \mathrm{~g} / \mathrm{L})$, sodium azide $(10 \mathrm{mg} / \mathrm{L})$ solution $(\mathrm{pH} 7.4)$. The antileptin serum was developed by rabbit immunization with leptin coupled to BSA. The detection range of the standard curve was $0.4-50 \mathrm{ng} / \mathrm{mL}$. Unknowns or standards $(100 \mu \mathrm{L})$ were incubated overnight at room temperature in the presence of $50 \mu \mathrm{L}$ of antileptin serum (final dilution of $1: 15,000$ ) and $50 \mu \mathrm{L}$ (approx 30,000 cpm) of tracer. Separation of bound and free hormone fractions was achieved by the addition first of $200 \mu \mathrm{L}$ of normal saline solution containing antirabbit $\gamma$-globulin and then $500 \mu \mathrm{L}$ of a solution of polyethylene glycol $6000(10 \%$ [wt/v] in normal saline) incubated for $30 \mathrm{~min}$ at room temperature before centrifuging at $3500 \mathrm{~g}$ for $30 \mathrm{~min}$ at $4^{\circ} \mathrm{C}$. Supernatants were aspirated and bound radioactivity was counted. The assay showed $2 \%$ and 0 crossreactivity with human leptin and mouse/rat anterior pituitary (luteinizing hormone, follicle-stimulating hormone; growth hormone; prolactin) hormones, respectively. The intra- and interassay CVs were 5-8 and 10$13 \%$, respectively.

\section{Statistical Analysis}

Results, expressed as mean \pm SEM, were analyzed by multifactorial analysis of variance followed by Fisher's test for comparison of different mean values (44).

\section{Acknowledgments}

We wish to thank Oscar Vercellini for excellent technical assistance and Susan H. Rogers for excellent editorial work. This work was supported by grants from the National (CONICET-PIP 4152/96; ANPCyT-PICT 5191/99) and Buenos Aires State (CICPBA) Research Councils, and UNLP of Argentina.

\section{References}

1. Fauzi, W., Herrera, G., Spiegelman, D., El Amin, A., Nestel, P., and Mohamed, K. (1997). Am. J. Clin. Nutr. 65, 1002-1009.

2. Zaman, K., Baqui, A., Yunus, M., Sack, R., Chowdhury, H., and Black, R. (1997). Acta Paediatr. 86, 923-927.

3. Jacobson, L., Zurakowski, D., and Majzoub, J. A. (1997). Endocrinology 138, 1048-1057.

4. Giovambattista, A., Chisari, A. N., Corró, L., Gaillard, R. C., and Spinedi, E. (2000). Neuroimmunomodulation 7, 92-98.

5. Suttmann, U., Selberg, O., Gallati, H., Ockenga, J., Deicher H., and Muller, M. J. (1994). Clin. Sci. 86, 461-467.

6. Doherty, J. F., Golden, M. H. N., Raynes, J. G., Griffin, G. E., and McAdam, K. P. W. J. (1993). Clin. Sci. 84, 169-175. 
7. Smith, S. R., Bledsoe, T., and Chhetri, M. K. (1975). J. Clin. Endocrinol. Metab. 40, 43-52.

8. Pimstone, B. (1976) Clin. Endocrinol. (Oxf.) 5, 79-95.

9. Bronson, F. H. (1986). Endocrinology 118, 2483-2487.

10. Gruaz, N. M., Pierroz, D. D., Rohner-Jeanrenaud, F., Sizonenko, P. C., and Aubert, M. L. (1993). Endocrinology 133, 1891-1894.

11. Hawkins, P., Steyn, C., McGarrigle, H. H., Saito, T., Ozaki, T., Stratford, L. L., Noakes, D. E., and Hanson, M. A. (2000). Exp. Physiol. 85, 85-96.

12. Alvarez, C., Martin, M. A., Goya, L., Bertin, E., Portha, B., and Pascual-Leone, A. M. (1997). Endocrinology 138, 2267-2273.

13. Wilson, M. R. and Hughes, S. J. (1997). J. Endocrinol. 154, 177-185.

14. Frederich, R. C., Hamann, A., Anderson, S., Lollman, B., Lowell, B. B., and Flier, J. S. (1995). Nat. Med. 1, 1311-1314.

15. Xu, B., Kalra, P. S., Farmerie, W. G., and Kalra, S. P. (1999). Endocrinology 140, 2868-2875.

16. Chisari, A. N., Giovambattista, A., Suescun, M., and Spinedi E. (1999). Proceedings from the 81 st Annual Meeting of the Endocrine Society, San Diego, CA (abstract).

17. Aird, F., Halasz, I., and Redei, E. (1997). Alcohol Clin. Exp. Res. 21, 1560-1566.

18. Cusin, I., Sainsbury, A., Doyle, P., Rohner-Jeanrenaud, F., and Jeanrenaud, B. (1995). Diabetes 44, 1467-1470.

19. Saladin, R., De Vos, P., Guerre-Millo, M., Leturque, A., Girard, J., Staels, B., and Auwerx, J. (1995). Nature 377, 527-529.

20. Dahri, S., Snoeck, A., Reusens-Billen, B., Remacle, C., and Hoet, J. J. (1991). Diabetes 40(Suppl. 2), 115-120.

21. Kalra, S. P., Dube, M. G., Pu, S., Xu, B., Horvath, T. L., and Kalra, P. S. (1999). Endocr. Rev. 20, 68-100.

22. Woods, S. C., Seeley, R. J., Porte, D. Jr., and Schwartz, M. W. (1998). Science 280, 1378-1382.

23. Picarel-Blanchot, F., Alvarez, C., Bailbe, D., Pascual-Leone, A. M., and Portha, B. (1995). Metabolism 44, 1519-1526.

24. McEwen, B. S. (1998). Ann. NY Acad. Sci. 840, 33-44.

25. Stokkan, K. A., Reiter, R. J., Vaughan, M. K., Nonaka, N. O., and Lerchl, A. (1991). Acta Endocrinol. (Copenh.) 125, 93-100.

26. Richard, D. (1993). Ann. NY Acad. Sci. 697, 155-172.
27. Vale, W., Spiess, J., Rivier, C., and Rivier, J. (1981). Science 213, 1394-1397.

28. Brady, L. S., Smith, M. A., Gold, P. W., and Herkenham, M. (1990). Neuroendocrinology 52, 441-447.

29. Timofeeva, E. and Richard, D. (1997). Neuroendocrinology 66, 327-340.

30. Bale, T. L., Contarino, A., Smith, G. W., Chan, R., Gold, L .H., Sawchenko, P. E., Koob, G. F., Vale, W. W., and Lee, K.-F. (2000). Nat. Genet. 24, 410-414.

31. Pazzoli, G., Bilezikjian, L. M., Perrin, M. H., Blount, A. L., and Vale, W. W. (1996). Endocrinology 137, 65-71.

32. Bradbury, M. J., McBurnie, M. I., Denton, D. A., Lee, K.-F., and Vale, W. W. (2000). Endocrinology 141, 2715-2724.

33. Antoni, F. A. (1993). Front. Neuroendocrinol. 14, 76-122.

34. Guillon, G., Trueba, M., Jourbet, D., Grazzini, E., Chouinard, L., Cote, M., Payet, M. D., Manzoni, O., Barberis, C., Robert, M., and Gallo-Payet, N. (1995). Endocrinology 136, 1285-1295.

35. Muller, M. B., Landgraf, R., Preil, J., Sillaber, I., Kresse, A. E., Keck, M. E., Zimmermann, S., Holsboer, F., and Wurst, W. (2000). Endocrinology 141, 4262-4269.

36. Tannenbaum, B. M., Tannenbaum, G. S., Brindley, D. N., Dallman, M. F., McArthur, M. D., and Meaney, M. J. (1997). Am. J. Physiol. 273, E1168-E1177.

37. Brindley, D. N., Cooling, J., Glenny, H. P., Burditt, S. L., McKechie, I. S. (1981). Biochem. J. 200, 275-284.

38. Nicholas, K. R. and Hartmann, P. E. (1991). Comp. Biochem. Physiol. 98A, 535-542.

39. Trottier, G., Koski, K. G., Brun, T., Toufexis, D. J., Richard, D., and Walker, C.-D. (1998). Endocrinology 139, 3704-3711.

40. Sapolsky, R. M., Romero, M., and Munck, A. U. (2000). Endocr. Rev. 21, 55-89.

41. Spinedi, E., Hadid, R., and Gaillard, R. C. (1997). Neuroendocrinology 66, 287-93.

42. Chisari, A., Spinedi, E., Voirol, M.-J., Giovambattista, A., and Gaillard, R. C. (1998). Endocrinology 139, 617-625.

43. Spinedi, E., Giacomini, M., Jacquier, M.w-C., and Gaillard, R. C. (1991). Neuroendocrinology 53, 160-170.

44. McElroy, W. D. and Swanson, C. P., eds. (1974). Biostatistical Analysis, Prentice-Hall, Englewood Cliffs, NJ. 